\title{
Reliability and Validity of the Beck Depression Inventory-II among Korean Adolescents
}

\author{
Eun-Ho Lee ${ }^{1 *}$, Soo-Ji Lee ${ }^{2 *}$, Soon-Taeg Hwang ${ }^{3}$, Sang-Hwang Hong ${ }^{4}$, and Ji-Hae Kim ${ }^{\bowtie}$ \\ 'Depression Center, Department of Psychiatry, Samsung Medical Center, Sungkyunkwan University School of Medicine, Seoul, Republic of Korea \\ ${ }^{2}$ Department of Psychiatry, Samsung Medical Center, Sungkyunkwan University School of Medicine, Seoul, Republic of Korea \\ ${ }^{3}$ Department of Psychology, Chungbuk National University, Cheongju, Republic of Korea \\ ${ }^{4}$ Department of Education, Chinju National University of Education, Jinju, Republic of Korea
}

Objective The Beck Depression Inventory-II (BDI-II) is one of the most popular scales for evaluating the severity of depression in adolescents as well as adults. The prevalence of depression increases during adolescence, and it has shown a rapid increase with occurrence at an earlier age and a tendency to continue into adulthood. Data from an adolescent nonclinical sample provides us more information related to depressive symptoms as potential risk factors. The current study was designed to two objectives: 1) to analyze the reliability and validity the BDI-II among Korean adolescents and 2) to evaluate the factorial structure in a Korean nonclinical adolescent sample.

Methods The participants included 1072 adolescent boys and girls. We assessed the internal consistency, corrected item-total correlation, and the convergent validity of the BDI-II. We also performed confirmatory factor analyses to determine the internal structure of the BDI-II for Korean adolescents using Mplus 6.1.

Results The Cronbach's alpha for the BDI-II total score was 0.89 . The correlation between the BDI-II and the PHQ-9 was strong $(\mathrm{r}=0.75)$, and anxiety-related measures were 0.68 and 0.71 , which were also in the high range. Among the five different factor structures, the modified three-factor model demonstrated the best overall fit.

Conclusion The BDI-II is a reliable tool for measuring the severity of depressive symptoms in Korean adolescents. Therefore, the findings can provide basic information for examining the prevalence rate, intervention strategies for depression in adolescents.

Psychiatry Investig 2017;14(1):30-36

Key Words Child and adolescence psychiatry, Psychometrics, Depressive disorder, Diagnosis.

\section{INTRODUCTION}

Depression has become a common disorder worldwide affecting a significant number of populations. ${ }^{1}$ The prevalence of depression increases during adolescence, and it has shown a rapid increase with occurrence at an earlier age and a tendency to continue into adulthood..$^{2-4}$ Depression during adolescence is associated with seriously negative outcomes such as increased risk for adolescents substance abuse, school dropout

Received: April 8, 2016 Revised: August 1, 2016

Accepted: August 30, 2016 Available online: December 27, 2016

$\triangle$ Correspondence: Ji-Hae Kim, PhD

Department of Psychiatry, Samsung Medical Center, Sungkyunkwan University School of Medicine, 81 Irwon-ro, Gangnam-gu, Seoul 06351, Republic of Korea

Tel: +82-2-3410-0931, Fax: +82-2-3410-0050

E-mail: jihae0931.kim@samsung.com

*These authors contributed equally to this work.

(c) This is an Open Access article distributed under the terms of the Creative Commons Attribution Non-Commercial License (http://creativecommons.org/licenses/by$\mathrm{nc} / 3.0$ ) which permits unrestricted non-commercial use, distribution, and reproduction in any medium, provided the original work is properly cited. and suicide attempts. ${ }^{3,5}$ Approximately $10-15 \%$ of all children and adolescents are currently experiencing depressive symptoms, ${ }^{6}$ with $2 \%$ of young children and $4-8 \%$ of adolescents suffering from Major Depressive Disorder (MDD). ${ }^{7}$ Harrington et al. reported that about sixty percent of the depressed adolescent group had one or more episodes of MDD in adulthood. ${ }^{8}$ In addition, a study performed in a community sample showed that people with subthreshold depression in adolescence had a similar prognosis to those who meet the criteria for major depression, and sample members with subthreshold depression are a group with elevated risks of later depression and suicide behaviors. ${ }^{9}$ If adolescent depression is not detected early and properly, there is a possibility that it may become chronic and may also become an obstacle to one's personal cognitive, emotional, and social functioning. ${ }^{2,3,5}$

The Beck Depression Inventory (BDI) is one of the most popular scales not only as one of the most widely used self-report instruments for evaluating the severity of depression, but 
also for screening for depression in clinical practice and general population of adolescents as well as adults. ${ }^{10}$ Since it was originally developed in English in 1961, the BDI has been translated into many languages with high levels of reliability and validity across cultures. ${ }^{11-14}$ The BDI has undergone two major revisions: in 1978 as the BDI-IA and in 1996 as the BDIII. ${ }^{1,15}$ In recent years, many attempts have been made to examine the psychometric properties of the BDI-II; however, these studies focused mostly on data from adult populations, ${ }^{16-19}$ and a limited number of reliability and validity studies of the BDI-II have been conducted in adolescents, either outpatients or inpatients. ${ }^{20,21}$

It is especially important to obtain a greater understanding of the influential results related to depressive symptoms as potential risk factors in nonclinical adolescents, given the fact that several mood-related disorders have their onset in childhood and adolescence. ${ }^{16}$ Data from a nonclinical sample are crucial for evaluating the response of subclinical patients who may potentially have mood symptoms or disorders. ${ }^{916}$ Also, given the high prevalence of depressive symptoms in the youth population, assessing the reliability and validity of the BDI-II provides significant background information for extending its use to population beyond a clinical sample.

There have been a few studies that revealed the factor structure of the BDI-II in an adolescent clinical sample, and it has been known that the factor structure of the BDI-II for adolescents might be different from that for adults. Steer and his colleagues identified a three-factor solution for the BDI-II in adolescent psychiatric outpatients. ${ }^{22}$ Another study conducted confirmatory factor analyses of several first-order solutions, but the analyses failed to provide adequate fit estimates for 408 adolescent psychiatric inpatients. ${ }^{20}$ Thus, conducting EFA, the model was composed of the two-factor solution, defined by cognitive-affective and somatic, unlike those of the BDI-II in an adult sample. ${ }^{15}$

Similar to the studies in a clinical adolescent sample, the factor structure of the BDI-II has not been established in a nonclinical adolescent sample. A research proposed the twofactor model of the BDI-II for Turkish nonclinical adolescents, defined by cognitive and somatic-affective. ${ }^{3}$ Also, bifactor analytic models, an alternative strategy for evaluating the structure of the BDI-II, were suggested in a sample of 414 U.S. adolescents. ${ }^{16}$ However, a study performed in Taiwanese nonclinical adolescents reported a modified three-factor model consisting of negative attitude, performance difficulty, and somatic factor. The author claimed that adding the three error correlations might be explained by the features of the samples. Adolescents in East Asia excessively value their academic performance and exams. When individuals have low academic performance, they devalue themselves, which may make them feel that there is no hope for the future. As they spend most of their time studying, they do not have time to enjoy other leisure activities. In addition, they frequently do not have enough time to sleep, which leads to changes in their appetite. ${ }^{23}$ On the other hand, the results in European and U.S. studies showed a different factor structure from the above test result in Taiwan. Therefore, it needs to be considered whether there is a cultural difference in adolescent depression.

The present study aims to investigate the psychometric properties of the BDI- II in a large and stratified sample of Korean nonclinical adolescents. It was designed to fulfill two main objectives: 1) to analyze the reliability and validity the BDI-II among Korean adolescents and 2) to evaluate the factorial structure in Korean nonclinical adolescents.

\section{METHODS}

\section{Participants}

The Korean Beck Anxiety and Depression Inventory (KBANDI) project is a cross-sectional, ongoing study designed to standardize and investigate the psychometric properties of the self-report measures (i.e., BDI-II, BAI, BHS) developed by Beck and colleagues from 5 regional areas (i.e., Seoul, Incheon, Cheongju, Daegu, Jinju) across South Korea. ${ }^{24}$ The current study used a subset of data drawn from the K-BANDI study. The participants included 1072 adolescent boys and girls who were recruited through education classes, recreational centers, advertisement, and via word-of-mouth. The mean age of the total sample was $15.35(\mathrm{SD}=1.73)$ years, wherein $51 \%$ of the sample were female. Parents were asked to sign the consent form and give the survey to their child to complete in a private place. The adolescent respondents received an approximately $5 \$$ reward for their participation. Completion of the survey was taken as a form of assent by the adolescents. All participants participated on a voluntarily and anonymous basis. This study was approved by the Samsung Medical Center Institutional Review Board.

\section{Measures}

\section{Beck Depression Inventory-II}

The BDI-II is a 21 -item self-report inventory designed to assess the presence and severity in depressive symptoms. Each item is rated on a 4-point Likert-type scale ranging from 0 to 3 , based on the severity in the last two weeks. The total score ranges from 0 to 63 , with higher scores indicating more severe depressive symptoms. With permission of the publisher, The Psychological Corporation, two independent licensed clinical psychologists (J.H.K. and S.T.H.) translated the original English version of the BDI-II into Korean and confirmed the 
content of the questionnaire through debate among three researchers. A proficient bilingual person who had a master's degree in clinical psychology re-translated it into English, and researchers reviewed and revised into its final version. The psychometric properties of the BDI-II among Korean adult population showed strong internal consistency, test-retest reliability and good concurrent and discriminant validity. And the bi-factor model showed the best fit among Korean adults. ${ }^{23}$

\section{Patient Health Questionnaire-9}

The PHQ-9 is a 9-item depression-screening instrument. ${ }^{25}$ It was developed based on the MDD criteria from the Diagnostic and Statistical Manual of Mental Disorders, Fourth edition (DSM-IV). Each item was rated based on the frequency of a depressive symptom in the past two weeks. The PHQ-9 score was calculated by assigning a score of $0,1,2$, and 3 , to the response categories of "not at all", "several days", "more than half the days", and "nearly every day", respectively. The Korean PHQ-9 showed good reliability and validity. ${ }^{26}$

\section{State-Trait Anxiety Inventory Korean YZ Form}

The STAI-KYZ assesses two types of anxiety with two scales: State Anxiety (STAI-S) and Trait Anxiety (STAI-T). State Anxiety is measured by 20 short descriptive statements, which the individual makes in reference to how he or she feels at the moment, whereas Trait Anxiety is measured by 20 statements that refer to one's general feelings. The Korean version of STAI showed good validity and reliability for adult samples. ${ }^{27}$

\section{Statistical analysis}

We used Mplus 6.1 to determine the factor structure of the BDI-II. All analyses were conducted using the mean and variance-adjusted weighted least squares estimation (WLSMV) method for the total adolescent sample. We tested the fitness of several models: 1) the one-factor model ("general depression"); 2) the model with two correlated factors ("cognitiveaffective" and "somatic"); 3) the model with three correlated first-order factors ("cognitive", "somatic-affective", and "guiltypunishment"); 4) the bifactor model, with all items loading on a general factor ("negative affect"), and two specific factors; 5) the model with three correlated factors ("negative attitude", "performance difficulty", and "somatic elements"). All error covariances and item cross-loadings described in the original

Table 1. Beck Depression Inventory-II for Korean adolescents item means, standard deviations, percentages symptomatic, and corrected item-total correlations $(\mathrm{N}=1072)$

\begin{tabular}{|c|c|c|c|c|}
\hline Symptom & M & $\mathrm{SD}$ & $\%$ & $\mathrm{r}_{\text {tot }}$ \\
\hline Sadness & 0.36 & 0.50 & 35.2 & 0.53 \\
\hline Pessimism & 0.51 & 0.65 & 43.8 & 0.52 \\
\hline Past failure & 0.39 & 0.61 & 32.8 & 0.54 \\
\hline Loss of pleasure & 0.40 & 0.58 & 35.6 & 0.49 \\
\hline Guilty feelings & 0.59 & 0.66 & 50.5 & 0.51 \\
\hline Punishment feelings & 0.34 & 0.64 & 27.1 & 0.50 \\
\hline Self-dislike & 0.34 & 0.68 & 22.9 & 0.63 \\
\hline Self-criticalness & 0.43 & 0.67 & 34.3 & 0.61 \\
\hline Suicidal thoughts or wishes & 0.34 & 0.52 & 32.3 & 0.50 \\
\hline Crying & 0.32 & 0.63 & 23.4 & 0.44 \\
\hline Agitation & 0.33 & 0.52 & 30.7 & 0.50 \\
\hline Loss of interest & 0.29 & 0.54 & 25.0 & 0.44 \\
\hline Indecisiveness & 0.54 & 0.62 & 47.5 & 0.47 \\
\hline Worthlessness & 0.24 & 0.54 & 19.3 & 0.60 \\
\hline Loss of energy & 0.47 & 0.62 & 39.6 & 0.56 \\
\hline Changes in sleeping pattern & 0.93 & 0.67 & 74.7 & 0.37 \\
\hline Irritability & 0.64 & 0.66 & 54.1 & 0.53 \\
\hline Changes in appetite & 0.78 & 0.72 & 62.2 & 0.34 \\
\hline Concentration difficulty & 0.53 & 0.66 & 44.0 & 0.50 \\
\hline Tiredness or fatigue & 0.70 & 0.60 & 63.4 & 0.53 \\
\hline Loss of interest in sex & 0.17 & 0.47 & 14.3 & 0.23 \\
\hline
\end{tabular}

$\%$ : total percentage endorsing response choices 1,2 , or $3, \mathrm{r}_{\text {tot }}$ : corrected item-total correlations 


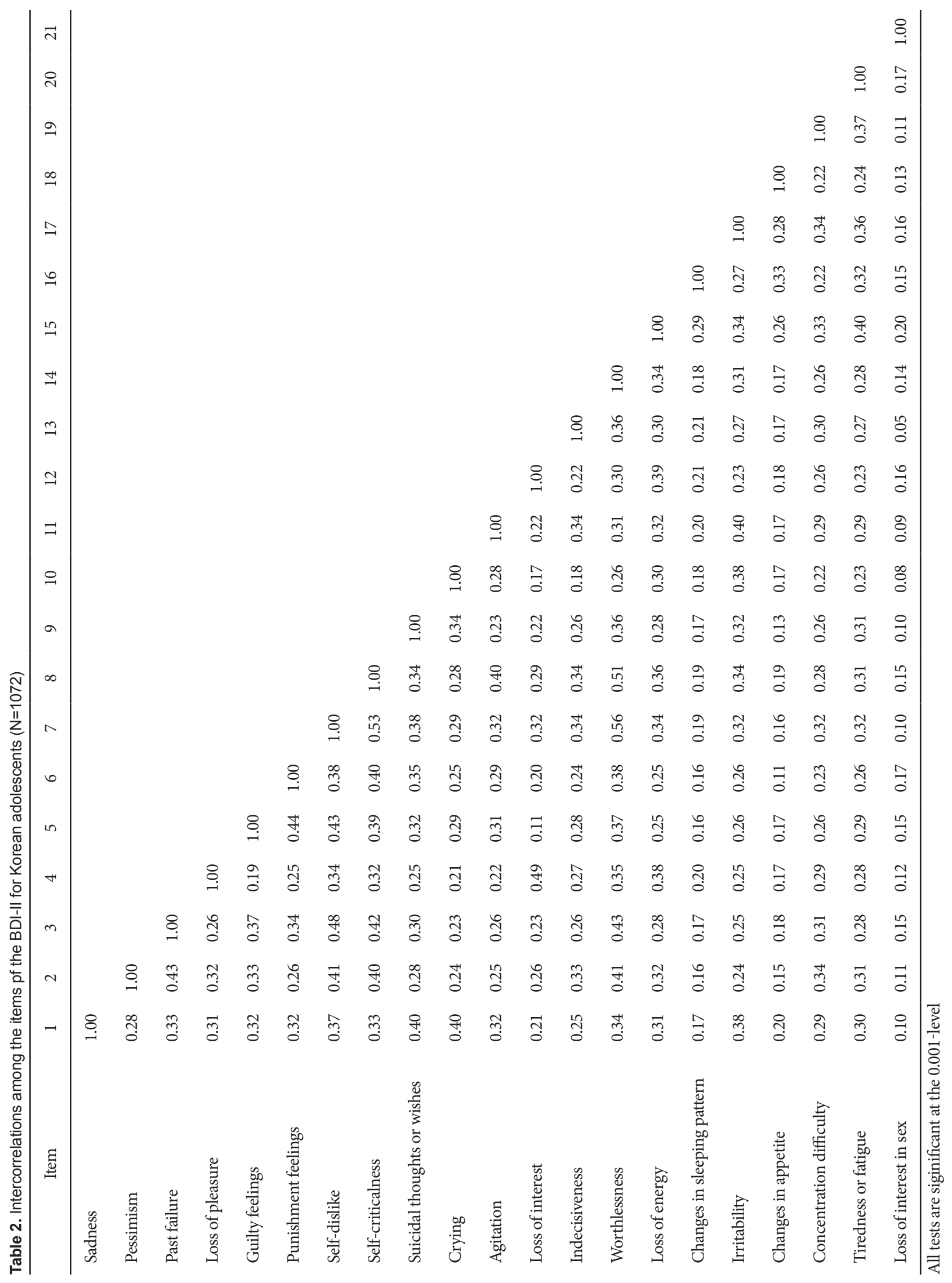


models were included in analyses. The fit of each model was assessed by means of the following three goodness of fit indices: 1 ) the Comparative Fit Index (CFI good fit $\geq 0.95$ ), 2) the Tucker-Lewis Fit Index (TLI good fit $\geq 0.95$ ), 3) the Root Mean Square Error of Approximation (RMSEA good fit $\leq 0.05)$ and its $90 \%$ confidence interval $(90 \% \mathrm{CI}){ }^{28}$

In order to investigate the item reliability of the Korean adolescents BDI-II items, we assessed their internal consistency using Cronbach alpha indices, corrected item-total correlations, and interitem correlations. To assess the convergent validity of the BDI-II, Pearson product-moment correlations with other self-report measures were calculated.

\section{RESULTS}

\section{Internal consistency}

Item means, standard deviations, percentages symptomatic, and corrected item-total correlations are summarized in Table 1. Cronbach's alpha estimates were computed to establish internal consistency of the BDI-II total. For the total sample of 1072 participants, the alpha estimate for the Korean BDI-II total scale score was 0.89 , which was comparable to that in previous studies. ${ }^{23}$ Examination of the corrected item-total correlation coefficients showed that each item contributed substantially to the total BDI-II score (range= 0.05 to 0.56 ). Item 21 (Loss of Interest in Sex) showed the lowest correlation with item 13 (Indecisiveness) (Table 2).

\section{Concurrent validity}

Using Pearson correlation analysis, we investigated the relations between scores on the BDI-II and the other self-report measures. The Pearson correlation coefficient for the Korean
BDI-II and the PHQ-9 was 0.75, indicating a strong correlation between these two measures of depression. Correlations between the Korean BDI-II and anxiety related measures (i.e., STAI-S, STAI-T) were 0.68 and 0.71 , which were also in the strong range (Table 4).

\section{Factorial validity}

We conducted a series of confirmatory factor analyses (CFA) to determine the factor structure of the Korean BDI-II. Several models were selected based on the previous findings using an adolescent sample. We examined one-, two-, three-, and modified three-factor models, and additionally evaluated a bifactor solution for the BDI-II. Results of the CFA are summarized in Table 3. Detailed descriptions of the models tested in the present study are as follows.

\section{Model A}

In this model, we constrained all 21 items to load onto a single factor. We tested this solution as a baseline model. The model failed to provide a good fit to the sample data $(\mathrm{CFI}=0.919$, TLI=0.910, RMSEA=0.065).

\section{Model B}

This model is based on the findings by Osman et al. ${ }^{20}$ They conducted a series of confirmatory factor analyses to establish factor structure of the BDI-II among adolescent psychiatric inpatients. This model is defined by two-correlated factors. The cognitive-affective factor was composed of items 1-10 and 12-14; and items 11 and 15-21 defined the somatic factor. This model did not provide an acceptable fit to the sample data $(\mathrm{CFI}=0.921, \mathrm{TLI}=0.912$, $\mathrm{RMSEA}=0.064)$.

Table 3. Summary of results from confirmatory factor analyses for Korean adolescent samples $(\mathrm{N}=1072)$

\begin{tabular}{clcccccc}
\hline Model & \multicolumn{1}{c}{ Study } & \multicolumn{1}{c}{$\chi^{2}$} & df & CFI & TLI & RMSEA & 90\% CI \\
\hline Model A & Null model & 1007.561 & 189 & 0.919 & 0.910 & 0.065 & $0.061-0.069$ \\
Model B & Osman et al. $^{24}$ & 988.849 & 188 & 0.921 & 0.912 & 0.064 & $0.060-0.068$ \\
Model C & Steer et al. $^{22}$ & 851.004 & 186 & 0.934 & 0.926 & 0.059 & $0.055-0.063$ \\
Model D & Osman et al. $^{16}$ & 575.060 & 168 & 0.960 & 0.950 & 0.048 & $0.044-0.053$ \\
Model E & Wu et al. $^{23}$ & 532.477 & 183 & 0.965 & 0.960 & 0.043 & $0.039-0.047$ \\
\hline
\end{tabular}

CFI: comparative fit index, TLI: Tucker-Lewis index, RMSEA: root mean square error of approximation, CI: confidence interval

Table 4. Means, standard deviations, and scale intercorrelations $(\mathrm{N}=1072)$

\begin{tabular}{ccccccc}
\hline Scale & M & SD & N & 1 & 2 & \\
BDI-II & 9.63 & 7.19 & 1071 & 1 & & \\
PHQ-9 & 3.67 & 3.68 & 1069 & $0.745^{*}$ & 1 \\
STAI-S & 37.48 & 10.99 & 1069 & $0.676^{*}$ & $0.599^{*}$ \\
STAI-T & 38.86 & 11.67 & 1069 & $0.707^{*}$ & $0.620^{*}$ & $0.907^{*}$ \\
\hline
\end{tabular}

*all tests are significant at the 0.001-level. BDI-II: Beck Depression Inventory-II, PHQ-9: Patient Health Questionnaire-9, STAI-S: State-Trait Anxiety Inventory-State, STAI-T: State-Trait Anxiety-Trait 


\section{Model C}

Steer et al. ${ }^{22}$ conducted an exploratory factor analysis to investigated factor structure of the BDI-II items using 210 adolescent outpatients. This model consisted of three positively correlated first-order factors: cognitive factor (items 2, 3, 7-9, 13,14 , and 19), somatic-affective factor (items 1, 4, 11, 12, 1518,20 , and 21), and guilty-punishment factor (items 5, 6, and $10)$. In the present study, we found that this model had a moderate but unsatisfactory fit to the sample data (CFI $=0.934$, TLI=0.926, RMSEA=0.059).

\section{Model D}

The Osman et al. ${ }^{16}$ nonclinical adolescent model consisted of three orthogonal first-order factors - a general depression factor (G), a somatic factor (items 15, 16, 18, 19, and 20), and a cognitive-affective factor (items $1-14,17$, and 21). This model provided a good fit to the sample data $(\mathrm{CFI}=0.960$, TLI=0.950, RMSEA=0.048). However, item 21 (Loss of Interest in Sex) failed to load onto the cognitive-affective factor. In addition, two items (Loss of Pleasure, Loss of Interest) showed negative relationships with the cognitive-affective factor. Based on these findings, we concluded that this model could not explain the internal structure of the BDI-II in our sample.

\section{Model E}

This model consisted of three oblique factors: negative attitude (items 1-3, 5-10, and 14), performance difficulty (items $4,11-13,17$, and 19), and somatic elements (items, 15, 16, 18,20 , and 21). Wu et al. ${ }^{23}$ investigated three different factor structures and modified the previously reported factor structure; three correlated item errors were set (item 2 , item 3 ; item 4 , item 12; item 16, item 18). This model provided best fit estimates for the sample data $(\mathrm{CFI}=0.965$, TLI $=0.960$, RM$\mathrm{SEA}=0.043$ ). The factor loadings ranged from values of 0.15 (item 21: Loss of Interest in Sex) to 0.73 (item 7: Self-Dislike). The correlations among the factors ranged from 0.75 to 0.94 .

\section{DISCUSSION}

The purpose of the present study was twofold: 1) to evaluate the reliability and validity and 2) to establish the factor structure of the BDI-II in a nonclinical population of Korean adolescents. The result of Cronbach alpha estimate was pretty high, comparable to those reported in Taiwanese and U.S. nonclinical adolescents. ${ }^{3,16}$ It was demonstrated that the BDI-II was internally consistent, and items were significantly correlated with the total score. Also, the mean of the Item 16 (changes in sleeping pattern) was 0.93 , which was the highest grade among all. The result was similar to the findings of the study in a U.S. nonclinical adolescent sample which reported that the mean of Item 16 was $1,{ }^{16}$ indicating that most adolescents who were not depressed or who were slightly depressed also experienced changed in sleep. Thus, it might be affected by hormones released during adolescence. ${ }^{29}$ In addition, the BDI-II showed a strong correlation with another depression-related measure; the correlation of the BDI-II with the PHQ-9 was strong, which are the instruments that measure the same construct (i.e., depression). ${ }^{25}$ Also, the STAI is known to include the items that reflect depression and general negative affect (i.e., dysphoric mood, negative self-appraisal) rather than pure anxiety, ${ }^{30,31}$ and the BDI-II and the STAI had moderately positive correlations as in the previous research. ${ }^{16}$

The present study tested five different model structures. The Models A, B, and C failed to provide an acceptable fit, and the Model D could not explain the internal structure of the BDI-II. As a result, our current findings revealed that the data for Korean adolescents are best represented by a modified three factors including negative attitude, performance difficulty, and somatic elements, supported by the previous research conducted in Taiwan. ${ }^{23}$ Unlike the two-factor model suggested by previous studies on nonclinical adolescents or students in Western countries, ${ }^{3,10}$ the three-factor structure with performance difficulty revealed by the research on nonclinical adolescents in East Asia may provide several possible explanations. One explanation could be that the Confucian values of East Asia put a great emphasis on education and academic achievement as a primary means for the individual to attain social status and self-fulfillment; hence, East Asian parents have enormous interest in their children's academic performance in high school and in the result of their children's college entrance exams. ${ }^{32,33}$ It occasionally makes Asian adolescents feel overwhelmingly pressured to perform well academically. ${ }^{23,34,35}$ As Asian adolescents spend most of their time studying, they may express their depressiveness through decline in academic achievement or difficulty in concentration.

Also, in the current study, the factor loading of item 21 (Loss of Interest in Sex) was 0.15, and this might be due to the characteristic of adolescent depression. A prior study with inpatient adolescent also reported item 21 showed low correspondence with DSM-IV depressive symptoms. ${ }^{20}$ Therefore, there might be possible that decreased libido may not be a typical feature of depression during adolescence; thus, it needs to be studied further in the different adolescent sample.

This study has some limitations. The present study did not conduct test-retest reliability; hence, we recommend that this issue should be studied further. Also, it is better to determine the factor structure of the BDI-II in clinical adolescents in order to compare with the results of this study.

In conclusion, the present research attempts to standardize the BDI-II with a relatively large sample of Korean adoles- 
cents. In addition, the reliability and validity of the BDI-II in this research corresponded with those in extant research. This finding reveals that the BDI-II is a reliable tool for measuring the severity of depressive symptoms in Korean adolescents. Therefore, the findings from this research can provide basic information for examining the prevalence rate, intervention strategies for depression in adolescents and so forth.

\section{Acknowledgments}

Portions of this research were presented at the semi-annual meeting of the Korean Clinical Psychological Association, Ilsan, October 2014.

\section{REFERENCES}

1. Wang YP, Gorenstein C. Psychometric properties of the Beck Depression Inventory-II: a comprehensive review. Rev Bras Psiquiatr 2013;35: 416-431.

2. Costello EJ, Copeland W, Angold A. Trends in psychopathology across the adolescent years: what changes when children become adolescents, and when adolescents become adults? J Child Psychol Psychiatry 2011; 52:1015-1025.

3. Uslu RI, Kapci EG, Oncu B, Ugurlu M, Turkcapar H. Psychometric properties and cut-off scores of the Beck Depression Inventory-II in Turkish adolescents. J Clin Psychol Med Settings 2008;15:225-233.

4. Aalto-Setala T, Marttunen M, Tuulio-Henriksson A, Poikolainen K, Lonnqvist J. Depressive symptoms in adolescence as predictors of early adulthood depressive disorders and maladjustment. Am J Psychiatry 2002; 159:1235-1237.

5. Subramaniam G, Harrell P, Huntley E, Tracy M. Beck Depression Inventory for depression screening in substance-abusing adolescents. J Subst Abuse Treat 2009;37:25-31.

6. Smucker MR, Craighead WE, Craighead LW, Green BJ. Normative and reliability data for the Children's Depression Inventory. J Abnorm Child Psychol 1986;14:25-39.

7. Birmaher B, Ryan ND, Williamson DE, Brent DA, Kaufman J, Dahl $\mathrm{RE}$, et al. Childhood and adolescent depression: a review of the past 10 years. Part I. J Am Acad Child Adolesc Psychiatry 1996;35:1427-1439.

8. Harrington R, Fudge H, Rutter M, Pickles A, Hill J. Adult outcomes of childhood and adolescent depression. I. Psychiatric status. Arch Gen Psychiatry 1990;47:465-473.

9. Fergusson DM, Horwood LJ, Ridder EM, Beautrais AL. Subthreshold depression in adolescence and mental health outcomes in adulthood. Arch Gen Psychiatry 2005;62:66-72.

10. Whisman MA, Perez JE, Ramel W. Factor structure of the Beck Depression Inventory-Second Edition (BDI-II) in a student sample. J Clin Psychol 2000;56:545-551.

11. Bosscher RJ, Koning H, Van Meurs R. Reliability and validity of the Beck Depression Inventory in a Dutch college population. Psychol Rep 1986;58:696-698.

12. Aalto AM, Elovainio M, Kivimaki M, Uutela A, Pirkola S. The Beck Depression Inventory and General Health Questionnaire as measures of depression in the general population: a validation study using the Composite International Diagnostic Interview as the gold standard. Psychiatry Res 2012;197:163-171.

13. Beck AT, Steer RA, Garbin MG. Psychometric properties of the Beck Depression Inventory: Twenty-Five years of evaluation. Clin Psychol Rev 1988;8:77-100.

14. Richter P, Werner J, Heerlein A, Kraus A, Sauer H. On the validity of the Beck Depression Inventory. A review. Psychopathology 1998;31: 160-168.

15. Beck AT, Steer RA, Brown GK. BDI-II: Beck Depression Inventory Manual. 2nd Ed. San Antonio, TX: Psychological Corporation; 1996.
16. Osman A, Barrios FX, Gutierrez PM, Williams JE, Bailey J. Psychometric properties of the Beck Depression Inventory-II in nonclinical adolescent samples. J Clin Psychol 2008;64:83-102.

17. Gomes-Oliveira MH, Gorenstein C, Lotufo Neto F, Andrade LH, Wang YP. Validation of the Brazilian Portuguese version of the Beck Depression Inventory-II in a community sample. Rev Bras Psiquiatr 2012;34:389-394.

18. Jaksic N, Ivezic E, Jokic-Begic N, Suranyi Z, Stojanovic-Spehar S. Factorial and diagnostic validity of the Beck Depression Inventory-II (BDI-II) in Croatian primary health care. J Clin Psychol Med Settings 2013;20:311-322.

19. Kuhner C, Burger C, Keller F, Hautzinger M. Reliability and validity of the Revised Beck Depression Inventory (BDI-II). Results from German samples. Nervenarzt 2007;78:651-656.

20. Osman A, Kopper BA, Barrios F, Gutierrez PM, Bagge CL. Reliability and validity of the Beck depression inventory-II with adolescent psychiatric inpatients. Psychol Assess 2004;16:120-132.

21. Arnarson TO, Olason DT, Smari J, Sigurethsson JF. The Beck Depression Inventory Second Edition (BDI-II): psychometric properties in Icelandic student and patient populations. Nord J Psychiatry 2008;62:360365.

22. Steer RA, Kumar G, Ranieri WF, Beck AT. Use of the Beck Depression Inventory-II with adolescent psychiatric outpatients. J Psychopathol Behav Assess 1998;20:127-137.

23. Wu PC, Huang TW. Gender-related invariance of the Beck Depression Inventory II for Taiwanese adolescent samples. Assessment 2014;21: 218-226.

24. Kim SG, Lee EH, Hwang ST, Hong SH, Lee KS, Kim JH. Reliability and valdity of the Korean version of the Beck Hopelessness Scale. J Korean Neuropsychiatr Assoc 2015;54:84-90.

25. Spitzer RL, Kroenke K, Williams JB. Validation and utility of a self-report version of PRIME-MD: the PHQ primary care study. Primary Care Evaluation of Mental Disorders. Patient Health Questionnaire. JAMA 1999;282:1737-1744.

26. Choi HS, Choi JH, Park KH, Joo KJ, Ga H, Ko HJ, et al. Standardization of the Korean version of Patient Health Questionnaire-9 as a Screening Instrument for Major Depressive Disorder. J Korean Acad Fam Med 2007;28:114-119.

27. Hahn DW, Lee CH, Chon KK, Spielberger CD. Korean Adaptation of the State-Trait Anxiety Inventory for Adults (STAI-KYZ). Seoul: Hakjisa; 2000.

28. Hu LT, Bentler PM. Cutoff criteria for fit indexes in covariance structure analysis: conventional criteria versus new alternatives. Struct Equ Modeling 1999;6:1-55.

29. Bailly D, Bailly-Lambin I, Querleu D, Beuscart R, Collinet C. Sleep in adolescents and its disorders. A survey in schools. Encephale 2004;30: 352-359.

30. Bieling PJ, Antony MM, Swinson RP. The State-Trait Anxiety Inventory, Trait version: structure and content re-examined. Behav Res Ther 1998; 36:777-788.

31. Caci H, Baylé FJ, Dossios C, Robert P, Boyer P. The Spielberger Trait Anxiety Inventory measures more than anxiety. Eur Psychiatry 2003;18: 394-400.

32. Lee M, Larson R. The Korean 'examination hell': long hours of studying, distress, and depression. J Youth Adolesc 2000;29:249-271.

33. Kwack YS, Shin JH. Factors related with suicidality in high-school students: focused on perceived academic performance. J Korean Acad Child Adolesc Psychiatry 2011;22:294-301.

34. Yi CC, Wu CI, Chang YH, Chang MY. The psychological well-being of Taiwanese youth: school versus family context from early to late adolescent. Int Sociol 2009;24:397-429.

35. Woo BS, Chang WC, Fung DS, Koh JB, Leong JS, Kee CH, et al. Development and validation of a depression scale for Asian adolescents. J Adolesc 2004;27:677-689. 